PROCEEDINGS OF THE

AMERICAN MATHEMATICAL SOCIETY

Volume 134, Number 5, Pages 1397-1404

S 0002-9939(05)08317-6

Article electronically published on October 7, 2005

\title{
ADJUGATES IN BANACH ALGEBRAS
}

\author{
ROBIN HARTE AND CARLOS HERNÁNDEZ
}

(Communicated by David R. Larson)

\begin{abstract}
A simple formula for the adjugate of a block triangle offers an alternative route to the determinant theory for Banach algebras.
\end{abstract}

Suppose $A$ is a complex linear algebra, with identity 1 and invertible group $A^{-1}$. The radical of $A$,

$$
\operatorname{Rad}(A)=\left\{a \in A: 1-A a \subseteq A^{-1},\right\}=\left\{a \in A: 1-a A \subseteq A^{-1}\right\},
$$

coincides with the intersection of the maximal left ideals, and also of the maximal right ideals. When

$$
\operatorname{Rad}(A)=\{0\}
$$

the algebra $A$ is described as semisimple. The spectrum of an element $a \in A$ is given by

$$
\sigma(a)=\sigma_{A}(a)=\left\{\lambda \in \mathbf{C}: a-\lambda \notin A^{-1}\right\} ;
$$

we shall also write

$$
\sigma^{\prime}(a)=\sigma_{A}^{\prime}(a)=\sigma(a) \backslash\{0\}
$$

for the nonzero spectrum. The nonzero spectrum offers a definition of "rank": we set $(11],[18])$

$$
\operatorname{rank}(a)=\operatorname{rank}_{A}(a)=\sup _{x \in A} \# \sigma^{\prime}(x a)=\sup _{x \in A} \# \sigma^{\prime}(a x) \leq \infty .
$$

If in particular $A$ is semisimple and a Banach algebra, then ([21, [19, 11])

$$
\operatorname{rank}(a) \leq 1 \Longleftrightarrow a A a \subseteq \mathbf{C} a .
$$

Thus, if $\operatorname{rank}(a) \leq 1$, then there is a bounded linear functional $\tau_{a} \in A^{*}$ for which

$$
L_{a} R_{a}=\tau_{a} \odot a: x \mapsto \tau_{a}(x) a .
$$

It is clear that if $a \neq 0$, then $\tau_{a}$ is uniquely determined; obviously we take $\tau_{0}=0$. Certainly if (0.7) holds, then

$$
x \in A \Longrightarrow \sigma(x a) \cup \sigma(x a) \subseteq\left\{0, \tau_{a}(x)\right\} .
$$

When $A$ is semisimple there are three equivalent definitions of the socle: the sum of the minimal left ideals, the sum of the minimal right ideals, and the linear subspace generated by the "rank one" elements. These in turn essentially reduce

Received by the editors December 10, 2004.

2000 Mathematics Subject Classification. Primary 46H05; Secondary 15A15.

Key words and phrases. Banach algebras, Fredholm theory, adjugate, determinant, socle.

The first author was supported by Enterprise Ireland Basic Research Grant SC/2002/0266.

(C)2005 American Mathematical Society 
to rank one idempotents. The socle $\operatorname{Soc}(A)$ provides the natural basis for Fredholm theory [4] in $A$, if we declare the "Fredholm" elements $a \in A$ to be those which are invertible modulo the socle:

$$
A_{\text {ess }}^{-1}=\left\{a \in A: a+\operatorname{Soc}(A) \in(A / \operatorname{Soc}(A))^{-1}\right\} .
$$

Since $([3,[18,6])$

$$
\operatorname{Soc}(A) \subseteq \bar{A} \equiv\{a \in A: a \in a A a\}
$$

the socle is a two-sided ideal consisting entirely of elements which have "generalized inverses", it turns out 8 that this "algebraic" concept of Fredholm element can also be made to look like the "spatial" version familiar when $A=B(X)$ consists of operators on a Banach space:

$$
A_{\text {ess }}^{-1}=\left\{a \in \bar{A}: L_{a}^{-1}(0) \cup R_{a}^{-1}(0) \subseteq \operatorname{Soc}(A)\right\} .
$$

Aupetit and Mouton [3] have noted how the socle also provides a basis for the extension to Banach algebras of the concepts of determinant and trace. In this note we offer a rather more pedestrian route to this determinant, using the Aupetit/Mouton formula for finite-dimensional algebras and extending by means of the "adjugate" or cofactor of suitable Banach algebra elements, together with a certain block triangle construction. We also extend the trace, and follow Murphy 20] in the use of "Fedosov's formula" to use it to reach the Fredholm index for elements invertible modulo the socle. The reason that the three kinds of socle coincide can be traced to an "orthogonalization" of projections (4, Lemma F.1.6]):

1. Lemma. If $p=p^{2} \in A$ and if $q=q^{2} \in \operatorname{Soc}(A)$, then there is $r=r^{2} \in \operatorname{Soc}(A)$ for which

$$
p A+q A=p A+r A \text { with } p r=0=r p .
$$

Proof. Since $(1-p) q \in \operatorname{Soc}(A) \subseteq \vec{A}$, there is $x \in A$ for which

$$
(1-p) q=r q \text { with } r=(1-p) x(1-p) q .
$$

Evidently

and if $a \in A$, then

$$
r^{2}=r \text { and } p r=0=r p
$$

$$
q a=p q a+r q a ; r a=-p q x(1-p) a+q x(1-p) a .
$$

We are of course being a little disingenuous in Lemma 1, since the orthogonalization is behind the proof ([3, Corollary 2.10]) of the regularity $(0.10)$. We might remark on a curious converse $\left([18,[6])\right.$ to $(0.10)$ : any ideal $J \subseteq{ }^{\top} A$ is a subset of the socle. This exactly parallels Smyth's observation 22 about ideals of algebraic elements. The essential ingredient in the Aupetit/Mouton recipe for the determinant and the trace is their concept of rank (0.5). If $\lambda \notin \operatorname{acc} \sigma(a)$ is at worst an isolated point of the spectrum of $a \in A$, then there is a Riesz projection

$$
\pi_{a}(\lambda)=\frac{1}{2 \pi i} \oint_{\lambda}(z-a)^{-1} d z .
$$

$1-\pi_{a}(\lambda)$ can be obtained from $a-\lambda$ by multiplying it by what is essentially its "Drazin inverse": Koliha [16] has noticed that $q=\pi_{a}(\lambda)$ can be characterized, with $c=a-\lambda$, by

$$
q=q^{2} ; q c=c q ; c+q \in A^{-1} ; 1+\mathbf{C} c q \subseteq A^{-1} .
$$


The Koliha-Drazin inverse and the spectral projection of course double commute with $a \in A$ : using either (1.3) or pure algebra ([7, Lemma 1])

$$
b v=v a \Longrightarrow \pi_{b}(\lambda) v=v \pi_{a}(\lambda) .
$$

2. Definition ([3]). If $A$ is a semisimple complex Banach algebra and $a \in \operatorname{Soc}(A)$, then

$$
\operatorname{det}(1+a) \equiv \operatorname{det}_{A}(1+a)=\prod_{\lambda \in \sigma(a)}(1+\lambda)^{\nu_{a}(\lambda)}
$$

and

$$
\operatorname{tr}(a) \equiv \operatorname{tr}_{A}(a)=\sum_{\lambda \in \sigma(a)} \lambda \nu_{a}(\lambda),
$$

where the multiplicity of $\lambda \in \sigma(a)$ is given by

$$
\nu_{a}(\lambda)=\operatorname{rank} \pi_{a}(\lambda) \equiv \operatorname{rank}_{A} \pi_{a}(\lambda) .
$$

The multiplicity (2.3) of $\lambda \in \sigma(a)$ is well defined, since

$$
a \in \operatorname{Soc}(A) \Longrightarrow \sigma(a)=\text { iso } \sigma(a) \text {. }
$$

Via Jacobson's lemma

$$
\operatorname{rank}(a b)=\operatorname{rank}(b a)
$$

for arbitrary $a, b \in A$, since only the nonzero spectrum $\sigma^{\prime}$ contributes to the determinant and the trace. Note that if $a \in A \subseteq B$, then

$$
\text { iso } \sigma_{A}(a) \subseteq \partial \sigma_{A}(a) \subseteq \sigma_{B}(a) \subseteq \sigma_{A}(a) .
$$

Thus in particular if $a \in \operatorname{Soc}(A)$, then its spectrum is unchanged when referred to a superalgebra $B \supseteq A$. However the formula (0.5) shows only that there is inequality

$$
\operatorname{rank}_{A}(a) \leq \operatorname{rank}_{B}(a) .
$$

Thus the determinant and the trace may change with the algebraic environment.

For a specific example embed the upper triangular $2 \times 2$ matrices via the left regular representation $a \mapsto L_{a}$ in the algebra $B$ of all $3 \times 3$ matrices: with

$$
\varphi(a)=u \text { and } \psi(a)=v \text { when } a=\left(\begin{array}{cc}
u & w \\
0 & v
\end{array}\right)
$$

we find

$$
\operatorname{det}_{B}\left(L_{a}\right)=\varphi(a) \operatorname{det}_{A}(a) ; \operatorname{tr}_{B}\left(L_{a}\right)=\varphi(a)+\operatorname{tr}_{A}(a) .
$$

If instead $B=A^{2}$ and $T a=(a, a)$, then

$$
\operatorname{det}_{B}(T a)=\operatorname{det}_{A}(a)^{2} ; \operatorname{tr}_{B}(T a)=2 \operatorname{tr}_{A}(a) .
$$

In a general semisimple Banach algebra the determinant and the trace are not defined for the same elements; the trace lives on the ideal $\operatorname{Soc}(A)$ while the determinant is defined for elements of the $\operatorname{coset} 1+\operatorname{Soc}(A)$. This would appear to rule out anything like a "Cayley-Hamilton theorem". If $a=a b a \in \operatorname{Soc}(A)$, then the products $q=a b$ and $p=b a$ are both idempotent and in the socle:

$$
q=q^{2}=a b \in \operatorname{Soc}(A) ; p=p^{2}=b a \in \operatorname{Soc}(A) ; a=a p=q a .
$$

Moreover if (2.11) holds, then

$$
q A q \subseteq \overparen{A} \text {, hence } q A q \subseteq \overparen{q A q},
$$


which by an old theorem of Kaplansky ([15, [23]) ensures that $q A q$ is finite dimensional:

$$
\operatorname{dim}(q A q)<\infty .
$$

Thus to reach an "adjugate" for elements $a \in 1+\operatorname{Soc}(A)$ we can begin by doing the job in the finite-dimensional algebra $q A q$, where the Cayley-Hamilton theorem does apply:

3. Definition. If $a \in A$ for a finite-dimensional algebra $A$ we define

$$
\operatorname{adj}(a) \equiv \operatorname{adj}_{A}(a)=\triangle_{a}^{A}(a) \equiv \triangle_{a}(a),
$$

where, identically on $\mathbf{C}$,

$$
\operatorname{det}_{A}(a-z) \equiv \operatorname{det}(a-z) \equiv \operatorname{det}(a)-z \triangle_{a}(z) .
$$

Here of course the determinant is given by the Aupetit/Mouton formula (2.1). Granted the determinant and the adjugate for finite dimensional algebras, it is an easy block triangularization to arrive at the general case:

4. Theorem. If $q=q^{2} \in A$, then

$$
A \leftrightarrow\left(\begin{array}{cc}
q A q & q A(1-q) \\
(1-q) A q & (1-q) A(1-q)
\end{array}\right) .
$$

$$
a=q a \in A \text { with } q=q^{2} \in \operatorname{Soc}(A),
$$

then

$$
\begin{gathered}
a \leftrightarrow\left(\begin{array}{cc}
a q & a(1-q) \\
0 & 0
\end{array}\right), 1+a \leftrightarrow\left(\begin{array}{cc}
q+a q & a(1-q) \\
0 & 1-q
\end{array}\right) \\
\operatorname{adj}_{A}(1+a) \leftrightarrow\left(\begin{array}{cc}
\operatorname{adj}_{q A q}(q+a q) & -\operatorname{adj}_{q A q}(q+a q) \cdot a(1-q) \\
0 & \operatorname{det}_{q A q}(q+a q) \cdot(1-q)
\end{array}\right), \\
\operatorname{det}_{A}(1+a)=\operatorname{det}_{q A q}(q+a q)
\end{gathered}
$$

and

$$
\operatorname{tr}_{A}(a)=\operatorname{tr}_{q A q}(a q)
$$

Proof. Generally if

$$
a=\left(\begin{array}{cc}
d & m \\
0 & b
\end{array}\right) \in A=\left(\begin{array}{cc}
D & M \\
0 & B
\end{array}\right),
$$

then $([12$, Theorem 6$])$

$$
\partial\left(\sigma_{D}(d) \cup \sigma_{B}(b)\right) \subseteq \sigma_{A}(a) \subseteq \sigma_{D}(d) \cup \sigma_{B}(b),
$$

so that if $d$ and $b$ have spectra consisting of isolated points,

$$
\sigma_{A}(a)=\sigma_{D}(d) \cup \sigma_{B}(b) \text { and } \sigma_{A}^{\prime}(a)=\sigma_{D}^{\prime}(d) \cup \sigma_{B}^{\prime}(b) \text {. }
$$

This means (0.5) that

$$
\operatorname{rank}_{A}(a)=\operatorname{rank}_{D}(d)+\operatorname{rank}_{B}(b) .
$$

This gives (4.5) and (4.6), and hence also (4.4) provided $1+a \in A$ is not a zero divisor. As observed by Kovacs/Silver/Williams ([17, [13]) we can embed the algebra $A$ in the ring $A[z]=A \otimes$ Poly of polynomials with $A$ coefficients, or consider the "Zarisky topology", to obtain (4.4) generally. 
Generally when (4.7) holds and $d \in 1+\operatorname{Soc}(D) \subseteq D, b \in 1+\operatorname{Soc}(B) \subseteq B$, then, writing

$$
\operatorname{adj}_{D}(d)=d^{\sim}, \operatorname{det}_{D}(d)=|d|, \operatorname{adj}_{B}(b)=b^{\sim}, \operatorname{det}_{B}(b)=|b|
$$

we find

$$
\operatorname{adj}_{A}(a)=\left(\begin{array}{cc}
|b| d^{\sim} & -d^{\sim} m b^{\sim} \\
0 & |d| b^{\sim}
\end{array}\right) \in A=\left(\begin{array}{cc}
D & M \\
N & B
\end{array}\right)
$$

If $a \in \operatorname{Soc}(A)$, then by (0.10) there is a projection $q=q^{2}$ for which (4.2) holds, namely $q=a b$ as in (2.11). It is clear from Theorem 4 that the right-hand sides of (4.4), (4.5) and (4.6) are independent of the particular $q=q^{2}$ satisfying (4.2). A direct proof uses the formula (4.11) twice:

\section{Theorem. Suppose}

$$
a=q a \text { with } q=q^{2}=r q \in \operatorname{Soc}(A) \text { and } r=r^{2} \in \operatorname{Soc}(A) .
$$

Then, with $B=r A r$ and $D=q A q$,

$$
\begin{gathered}
\left(\begin{array}{cr}
\operatorname{adj}_{D}(q+a q) & -\operatorname{adj}_{D}(q+a q) \cdot a(1-q) \\
0 & \operatorname{det}_{D}(q+a q) \cdot(1-q)
\end{array}\right) \\
\leftrightarrow\left(\begin{array}{cc}
\operatorname{adj}_{B}(r+a r) & -\operatorname{adj}_{B}(r+a r) \cdot a(1-r) \\
0 & \operatorname{det}_{B}(r+a r) \cdot(1-r)
\end{array}\right) \\
\operatorname{det}_{D}(q+a q)=\operatorname{det}_{B}(r+a r)
\end{gathered}
$$

and

$$
\operatorname{tr}_{D}(a q)=\operatorname{tr}_{B}(a r)
$$

Proof. If, generalizing (4.7),

$$
a=\left(\begin{array}{ccc}
d & m & k \\
0 & c & \ell \\
0 & 0 & b
\end{array}\right) \in\left(\begin{array}{ccc}
D & M & K \\
M^{\prime} & C & L \\
K^{\prime} & L^{\prime} & B
\end{array}\right)
$$

then in two different ways, applications of (4.11) give, writing $\operatorname{adj}_{B}(b)=b^{\sim}$ and $\operatorname{det}_{D}(d)=|d|$, etc.,

$$
\operatorname{adj}_{A}\left(\begin{array}{ccc}
d & m & k \\
0 & c & \ell \\
0 & 0 & b
\end{array}\right)=\left(\begin{array}{ccc}
|c||b| d^{\sim} & -d^{\sim} m|b| c^{\sim} & d^{\sim}\left(m c^{\sim} \ell-k|c|\right) d^{\sim} \\
0 & |a||b| c^{\sim} & -|d| c^{\sim} \ell b^{\sim} \\
0 & 0 & |d||c| b^{\sim}
\end{array}\right) .
$$

This would give (5.2) provided there was inclusion $q A q \subseteq r A r$, which would need commutivity $q r=r q$. Note however that if (5.1) holds, then $q r$ and $(1-q) r$ are both idempotent; thus

$$
a \in q r A q \subseteq r A r_{\cap} q A q .
$$

We may therefore apply (5.5) twice, with $D=q r A q$ and $\left(\begin{array}{cc}D & M \\ M^{\prime} & C\end{array}\right)$ either $q A q$ or $r A r$.

If $a \in A$ is Fredholm with respect to the socle, in the sense (0.9), then there are $b^{\prime}, b^{\prime \prime} \in A$ for which

$$
\left\{1-b^{\prime} a, 1-a b^{\prime \prime}\right\} \subseteq \operatorname{Soc}(A) ;
$$

necessarily $b^{\prime \prime}-b^{\prime} \in \operatorname{Soc}(A)$. In view of $(0.10)$ we can arrange that $b^{\prime \prime}=b^{\prime}=b$ with $a=a b a$. Following Murphy [20] we can therefore make the following definition. 
6. Definition. If $a \in A$ is Fredholm and $b^{\prime}, b^{\prime \prime}$ satisfy (5.7), then

$$
\operatorname{index}(a)=\operatorname{tr}\left(a b^{\prime \prime}-b^{\prime} a\right) .
$$

To see that (6.1) is independent of the particular choice of $b^{\prime}, b^{\prime \prime}$ satisfying (5.7), we need to know that

$$
a \in A, d \in \operatorname{Soc}(A) \Longrightarrow \operatorname{tr}(a d-d a)=0 .
$$

While this probably follows from the proof of Theorem 3.3 of Aupetit/Mouton [3], we can see it here first for finite-dimensional algebras and then generally, using the argument of Theorem 3 above. Alternatively it is the Jacobson lemma that $\sigma^{\prime}(a d)=\sigma^{\prime}(d a)$, and we just have to check (cf. (1.5); [6], Lemma 2)) that for each point $\lambda \in \sigma^{\prime}(a d)$ there is equal multiplicity

$$
\nu_{d a}(\lambda)=\nu_{a d}(\lambda) .
$$

As noted by Murphy [20, the index enjoys familiar properties:

7. Theorem. If $a \in A$ is Fredholm, then

$$
\operatorname{index}(a+d)=\operatorname{index}(a) \text { if } d \in \operatorname{Soc}(A),
$$

and, if $b^{\prime}, b^{\prime \prime}$ satisfy (5.7),

$$
\operatorname{index}\left(b^{\prime}\right)=\operatorname{index}\left(b^{\prime \prime}\right)=-\operatorname{index}(a) .
$$

If $a, a^{\prime}$ are Fredholm, then the logarithmic law holds:

$$
\operatorname{index}\left(a^{\prime} a\right)=\operatorname{index}\left(a^{\prime}\right)+\operatorname{index}(a) .
$$

The index is locally constant: if $\{1-b a, 1-a b\} \subseteq \operatorname{Soc}(A)$, then

$$
1+\left(a^{\prime}-a\right) b \in A^{-1} \Longrightarrow \operatorname{index}\left(a^{\prime}\right)=\operatorname{index}(a) .
$$

Proof. Most of this is word for word as in Murphy [20]. For (7.1) we argue (20], Theorem 2.3) that

$$
\text { index }(a+d)=\operatorname{tr}((a+d) b-b(a+d))=\operatorname{tr}(a b-b a)=\operatorname{index}(a)+0 .
$$

For (7.3) we argue ([20], Theorem 2.4) that

$$
\begin{aligned}
\operatorname{index}\left(a^{\prime} a\right) & =\operatorname{tr}\left(a^{\prime} a b b^{\prime}-b b^{\prime} a^{\prime} a\right) \\
& =\operatorname{tr}\left(a^{\prime}(1+d) b^{\prime}-b^{\prime} a^{\prime}+b^{\prime} a^{\prime}-b b^{\prime} a^{\prime} a\right) \\
& =\operatorname{tr}\left(a^{\prime} b^{\prime}-b^{\prime} a^{\prime}\right)+\operatorname{tr}\left(a^{\prime} d b^{\prime}\right)+\operatorname{tr}\left(b^{\prime} a^{\prime}-b b^{\prime} a^{\prime} a\right) \\
& =\operatorname{index}\left(a^{\prime}\right)+\operatorname{tr}\left(b^{\prime} a^{\prime}(1+d)-b b^{\prime} a^{\prime} a\right) \\
& =\operatorname{index}\left(a^{\prime}\right)+\operatorname{tr}\left(\left(b^{\prime} a^{\prime} a\right) b-b\left(b^{\prime} a^{\prime} a\right)\right) \\
& =\operatorname{index}\left(a^{\prime}\right)+\operatorname{index}\left(b^{\prime} a^{\prime} a\right) \\
& =\operatorname{index}\left(a^{\prime}\right)+\operatorname{index}\left(\left(1+d^{\prime}\right) a\right)
\end{aligned}
$$

with $d=1-a b$ and $d^{\prime}=1-b^{\prime} a^{\prime}$ in $\operatorname{Soc}(A)$. For (7.4) we argue ([20, Theorem 2.5) that

$c=1+\left(a^{\prime}-a\right) b \in A^{-1} \Longrightarrow 0=\operatorname{index}\left(a^{\prime} b-d\right)=\operatorname{index}\left(a^{\prime} b\right)=\operatorname{index}\left(a^{\prime}\right)+\operatorname{index}(b)$,

giving index $\left(a^{\prime}\right)=-\operatorname{index}(b)=\operatorname{index}(a)$. Finally (7.4) also gives (7.2). 
Since evidently the socle and its scalar perturbations are contained in the closure of the invertibles, it is clear ([9], [10, (7.3.4.4)) that

$$
A^{-1}+\operatorname{Socle}(A) \subseteq \widetilde{A} \equiv\left\{a \in A: a \in a A^{-1} a\right\} .
$$

We thus obtain a familiar decomposition:

8. Theorem. If $a \in A$ the following two conditions are equivalent:

$$
\begin{gathered}
a \in A^{-1}+\operatorname{Soc}(A), \\
a \in \widetilde{A}_{\cap} A_{\text {ess }}^{-1} .
\end{gathered}
$$

Each of them imply

$$
a \in A_{\text {ess }}^{-1} \text { with } \operatorname{index}(a)=0 .
$$

Proof. For implication (8.1) $\Longrightarrow(8.2)$ argue

$$
a=c^{-1}+d \in A^{-1}+\operatorname{Soc}(A) \Longrightarrow c a \in 1+\operatorname{Soc}(A) \subseteq \breve{A} \cap A_{\text {ess }}^{-1} .
$$

Conversely if (8.2) holds, then ( 8 ] Theorem 2.2; 10] Theorem 7.8.2), using (0.11), there is $b \in A$ for which

$$
b \in A^{-1} ; 1-b a \in \operatorname{Soc}(A) ; 1-a b \in \operatorname{Soc}(A) .
$$

Now $b^{-1}-a=b^{-1}(1-b a) \in \operatorname{Soc}(A)$. For implication $(8.2) \Longrightarrow(8.3)$ argue

$$
\begin{aligned}
& a=a b a \in A_{\text {ess }}^{-1} \text { with } b \in A^{-1} \\
& \quad \Longrightarrow \operatorname{index}(a)=\operatorname{index}(a)+\operatorname{index}(b)+\operatorname{index}(a)=2 \operatorname{index}(a) .
\end{aligned}
$$

The condition (8.3) is in general weaker than (8.1) and (8.2): for example take

$$
a=\left(\begin{array}{cc}
b & 0 \\
0 & b^{\prime}
\end{array}\right) \in\left(\begin{array}{cc}
B & 0 \\
0 & B
\end{array}\right) \text { with } \operatorname{index}\left(b^{\prime}\right)=1=-\operatorname{index}(b) .
$$

The socle can be extended to the larger ideal of "inessential" elements,

$$
\text { Hull } \operatorname{Soc}(A)=\{d \in A: d+\operatorname{Soc}(A) \in \operatorname{Rad}(A / \operatorname{Soc}(A))\},
$$

which gives the same "Fredholm theory":

9. Theorem. If $a \in A$, then

$$
a+\operatorname{Hull} \operatorname{Soc}(A) \in(A / \operatorname{Hull} \operatorname{Soc}(A))^{-1} \Longrightarrow a \in A_{\text {ess }}^{-1} .
$$

There is inclusion

$$
A^{-1}+\operatorname{Hull} \operatorname{Soc}(A) \subseteq \bar{A}
$$

and implication

$$
d \in \text { Hull } \operatorname{Soc}(A) \Longrightarrow \operatorname{index}(a+d)=\operatorname{index}(a) .
$$

Proof. The implication (9.1) holds ([10, Theorem 7.2.6; 8 , (2.5.11)) separately for left and for right invertibility: if $a \in A$ is left invertible modulo the Hull of the socle, then there is $b \in A$ for which $1-b a \in \operatorname{Hull} \operatorname{Soc}(A)$ and hence $c \in A$ for which

$$
1-c b a=1-c(1-(1-b a)) \in \operatorname{Soc}(A) .
$$

Inclusion (9.2) follows easily ([10], Theorem 7.3.3; 8$]$, (2.5.12)), using lemmas of Atkinson. For (9.3) suppose $d \in \operatorname{Hull} \operatorname{Soc}(A)$. Then $1-A d \cup 1-d A \subseteq A_{\text {ess }}^{-1}$, and if $\{1-a b, 1-b a\} \subseteq \operatorname{Soc}(A)$, then there are $b^{\prime}, b^{\prime \prime} \in A$ for which

$$
\left\{b^{\prime}(1-b d),(1-d b) b^{\prime}, b^{\prime \prime}\left(1+b^{\prime} b d\right),\left(1+d b b^{\prime}\right) b^{\prime \prime}\right\} \subseteq \operatorname{Soc}(A),
$$


giving

index $(a+d)=\operatorname{tr}((a+d) b-b(a+d))=\operatorname{tr}(a b-b a)+\operatorname{tr}(d b-b d)=\operatorname{index}(a)+\operatorname{tr}\left(b^{\prime \prime}-b^{\prime}\right)$.

\section{REFERENCES}

1. B. Aupetit, A primer on spectral theory, Springer-Verlag 1991. MR.1083349 (92c:46001)

2. B. Aupetit, Propriétés spectrales des algébres de Banach, Springer-Verlag 1979. MR0549769 (81i:46055)

3. B. Aupetit and H. du T. Mouton, Trace and determinant in Banach algebras, Studia Math. 121 (1996) 115-136. MR1418394 (97i:46086)

4. B.A. Barnes, G.J. Murphy, M.R.F. Smyth and T.T. West, Riesz and Fredholm theory in Banach algebras, Pitman 1982. MR0668516 (84a:46108)

5. M. Bresar and P. Semrl, Finite rank elements in Banach algebras, Studia Math. 128 (1998) 287-298. MR1611128(99a:46089)

6. R.M. Brits, L. Lindeboom and H. Raubenheimer, On ideals of generalized invertible elements in Banach algebras, Proc. Royal Irish Acad. (to appear).

7. B.P. Duggal, R.E. Harte and I.H. Jeon, Polaroid operators and Weyl's theorem, Proc. Amer. Math. Soc. 132 (2004) 1345-1349. MR 2053338 (2004m:47005)

8. R.E. Harte, Fredholm, Weyl and Browder theory, Proc. Royal Irish Acad. 85A (1985) 151-176. MR.0845539 (87h:47029)

9. R.E. Harte, Regular boundary elements, Proc. Amer. Math. Soc. 88 (1987) 328-330. MR0870795 (88d:46088)

10. R.E. Harte, Invertibility and singularity, Dekker 1988. MR0920812 (89d:47001)

11. R.E. Harte, On rank one elements, Studia Math. 117 (1995) 73-77. MR1367694 (96i:46055)

12. R.E. Harte, Block diagonalization in Banach algebras, Proc. Amer. Math. Soc. 129 (2001) 181-190. MR1784022 (2001k:47013)

13. R.E. Harte, C. Hernández and E. de Oteyza, Adjugates of commuting block matrices, (preprint).

14. R.E. Harte and H. Raubenheimer, Fredholm, Weyl and Browder theory III, Proc. Royal Irish Acad. 95A 1995 11-16. MR.1369040(96i:47002)

15. I. Kaplansky, Regular Banach algebras, Jour. Indian Math. Soc. 12 (1948) 57-62. MR0029106 $(10: 549 \mathrm{~b})$

16. J.J. Koliha, A generalized Drazin inverse, Glasgow Math. Jour. 38 (1996) 367-381. MR.1417366 (98b:46065)

17. I. Kovacs, D.S. Silver and S.G. Williams, Determinants for commuting block matrices, Amer. Math. Monthly 106 (1999) 950-952. MR1732497

18. L. Lindeboom and H. Raubenheimer, On regularities and Fredholm theory, Czech Math. Jour. 52 (2002) 565-574. MR1923262(2003h:46070)

19. H. du T. Mouton and H. Raubenheimer, On rank one and finite elements in Banach algebras, Studia Math. 104 (1993) 17-25. MR.1220661 (94c:46095)

20. G.J. Murphy, Fredholm index theory and the trace, Proc. Royal Irish Acad. 94A (1994) 161166. MR1369029 (96m:47021)

21. J. Puhl, The trace of finite and nuclear elements in Banach algebras, Czech Math. Jour. 28 (1978) 656-676. MR0506439 (81a:47024)

22. M.R.F. Smyth, Riesz theory in Banach algebras, Math. Zeit. 45 (1975) 145-155. MR0394210 $(52: 15013)$

23. A.W. Tullo, Conditions on Banach algebras which imply finite dimensionality, Proc. Edinburgh Math. Soc. 20 (1976) 1-5. MR0415318 (54:3407)

School of Mathematics, Trinity College, Dublin 2, Ireland

E-mail address: rharte@maths.tcd.ie

Instituto de Matemáticas, Universidad Nacional Autónoma de México, México, D.F. 04510, MÉxico

E-mail address: carlosh@servidor.unam.mx 